\title{
Efeitos de borda sobre a vegetação e estruturação populacional em fragmentos de Cerradão no Sudoeste Goiano, Brasil
}

\author{
Matheus de Souza Lima-Ribeiro ${ }^{1}$
}

Recebido em 4/12/2006. Aceito em 8/08/2007

\begin{abstract}
RESUMO - (Efeitos de borda sobre a vegetação e estruturação populacional em fragmentos de Cerradão no Sudoeste Goiano, Brasil). Efeitos de borda são modificações nos parâmetros físicos, químicos e biológicos observadas na área de contato do fragmento de vegetação com a matriz circundante. O objetivo desse estudo foi testar a hipótese de que os efeitos de borda afetam alguns parâmetros vegetacionais e populacional em fragmentos de Cerradão no município de Caiapônia, Goiás. Para isso, estudou-se a estrutura da vegetação, o padrão de distribuição espacial de Vernonia aurea Mart. ex DC. (Asteraceae) e as condições microclimáticas (temperatura do ar e do solo e umidade relativa do ar) em dois ambientes, borda e interior, de três fragmentos de Cerradão com diferentes tamanhos (1,5 ha; 9 ha e 35 ha). Os resultados mostraram uma nítida influência dos efeitos de borda em todos os parâmetros analisados, uma vez que a estrutura da vegetação, o nível de agregação dos indivíduos de $V$. aurea e os parâmetros microclimáticos foram diferentes entre o interior e a borda dos fragmentos analisados. Esses dados corroboram com outros estudos realizados em diferentes ecossistemas, indicando que a fragmentação dos habitats modifica a composição e estrutura da vegetação, a estrutura espacial e a dinâmica das populações vegetais e, conseqüentemente, animais, podendo levar à extinção de populações locais e redução da diversidade biológica.
\end{abstract}

Palavras-chave: cerrado, fragmentação de habitat, efeitos de borda, estrutura vegetacional, distribuição espacial

ABSTRACT - (Edge effects on vegetation and population structure in Cerradão fragments of Southwest Goiás, Brazil). Edge effects are modifications in the physical, chemical and biological parameters observed in areas of contact of forest fragment with the surrounding environment. This paper aimed to test the hypothesis that the edge effects influence some vegetation and population parameters in Cerradão fragments in Caiapônia County, Goiás. To this effect, vegetation structure, Vernonia aurea Mart. ex DC. (Asteraceae) spatial distribution and microclimate conditions (air and soil temperature and air humidity) were analyzed in two habitats, edge and interior, of three Cerradão fragments with different areas (1.5 ha, 9 ha, and $35 \mathrm{ha})$. The results showed a strong influence of edge effects on all parameters analyzed, that is, vegetation structure, $V$. aurea aggregation index and microclimate parameters were all different between interior and edge habitats. These results corroborate other studies conducted in different ecosystems, indicating that habitat fragmentation modifies vegetation structure and composition, plant population dynamics and spatial structure, and as a result, local biological diversity is reduced.

Key words: brazilian savanna, habitat fragmentation, edge effects, vegetation structure, spatial distribution

\section{Introdução}

O Cerrado, no Planalto Central brasileiro, vem sofrendo constantes degradações em seus ecossistemas naturais devido às ações antrópicas como agricultura e pecuária que, pelo modelo atual de desenvolvimento, necessitam da retirada da vegetação natural para a implantação de pastagens e campos agrícolas, compostos, principalmente, por monoculturas (Diniz 1984; Sala et al. 2000). A substituição de grandes áreas de vegetação natural por ecossistemas diferentes, como pastagens e campos agrícolas, leva à criação de fragmentos isolados, imersos em uma matriz antrópica (Forman \& Godron 1986; Franklin 1993). A criação de fragmentos implica na formação de uma borda florestal, definida como uma região de contato entre a área ocupada (matriz antrópica) e o fragmento de vegetação natural (Williams-Linera et al. 1997; Primak \& Rodrigues 2001), promovendo alteração nos parâmetros físicos, químicos e biológicos do sistema, como disponibilidade energética e fluxo de organismos entre tais ambientes (Wiens et al. 1993). De maneira geral, estas modificações nas áreas mais externas dos fragmentos florestais, geradas pelo contato com a matriz, são chamadas "efeitos de borda" (Murcia 1995; Primak \& Rodrigues 2001).

\footnotetext{
Universidade Federal de Goiás, Centro de Ciências Agrárias e Biológicas, Departamento de Biologia, Campus de Jataí, BR 364, Km 192, Setor Industrial, 75800-000 Jataí, GO, Brasil (paleo_ribeiro@yahoo.com.br)
} 
Os efeitos de borda são divididos em dois tipos: abióticos ou físicos e os biológicos diretos e indiretos (Murcia 1995). Os efeitos abióticos envolvem mudanças nos fatores climáticos ambientais, onde a zona de influência das bordas apresenta maior exposição aos ventos, altas temperaturas, baixa umidade e alta radiação solar (ver exemplos em DaviesColley et al. 2000; Redding et al. 2003). Os efeitos biológicos diretos envolvem mudanças na abundância e na distribuição de espécies provocados pelos fatores abióticos nas proximidades das bordas, como por exemplo, o aumento da densidade de indivíduos devido à maior produtividade primária causada pelos altos níveis de radiação solar (ver exemplos em MacDougall \& Kellman 1992; Didhan \& Lawton 1999). Os indiretos envolvem mudanças na interação entre as espécies, como predação, parasitismo, herbivoria, competição, dispersão de sementes e polinização (ver exemplos em Galetti et al. 2003; Kollmann \& Buschor 2003).

Todo este processo acaba sendo um fator selecionador das comunidades capazes de se instalar e utilizar as bordas como área de desenvolvimento, devido às adaptações necessárias para as espécies habitarem com sucesso esses ambientes ecotonais (Willson \& Crome 1989; Malcolm 1994). A distribuição das espécies num ambiente reflete o nível de adaptabilidade frente às diversas pressões seletivas, uma vez que devem existir condições essenciais à sobrevivência para que ocorra a ocupação e a colonização de determinado local (Ridley 2006; Rizzini 1997). Desta maneira, o estudo da composição florística e da distribuição espacial de populações biológicas fornece informações importantes para a compreensão dos fatores bióticos e abióticos determinantes da estruturação populacional e das interações ecológicas ali existentes (Fernandes 1998).

Em uma população qualquer, os indivíduos podem apresentar três padrões básicos de distribuição espacial: aleatório, agrupado e uniforme. O padrão aleatório, em que a localização de um indivíduo não interfere na localização de outro da mesma espécie, se opõem ao padrão de distribuição agrupado (ou agregado), onde a presença de um recurso (como tipo de solo, luminosidade) tem alta probabilidade de influenciar a localização dos indivíduos de uma mesma espécie, apresentando assim, baixos índices de dispersão. No padrão de distribuição uniforme (ou regular), a população apresenta alto índice de dispersão e os indivíduos possuem distâncias semelhantes uns dos outros (Greig-Smith 1964; Kershaw 1973; MuellerDombois \& Ellenberg 1974; Barbour et al. 1987).
Apesar da possibilidade de uma população apresentar distribuição uniforme, as plantas muito raramente se distribuem dessa maneira na natureza. Diferentes condições ambientais e disponibilidade de recursos, bem como distúrbios naturais ou antrópicos, são alguns dos fatores que influenciam o padrão espacial e a dinâmica das populações vegetais (Lundberg \& Ingvarsson 1998; Leite 2001). Estes fatores não somente modificam o padrão de distribuição e abundância, como também influenciam a taxa de crescimento, produção de sementes, área foliar, comprimento das raízes e tamanho dos indivíduos (Greig-Smith 1964; Kershaw 1973; Mueller-Dombois \& Ellenberg 1974; Barbour et al. 1987).

As possíveis causas nas diferenças florísticas e estruturais (distribuição espacial das populações, por exemplo) existentes entre a borda e o interior de fragmentos podem ser esclarecidas pelo estudo de suas condições microclimáticas. Não obstante, parece não haver um padrão muito claro sobre até que ponto as alterações microclimáticas das bordas podem ser percebidas no interior dos fragmentos (Kapos et al. 1997; Nascimento \& Laurance 2006). Embora exista conhecimento relativamente vasto sobre os efeitos de borda, ainda é muito difícil prever a trajetória dos processos ecológicos em bordas assim como as transformações nos padrões naturais (Rodrigues \& Nascimento 2006).

Os efeitos de borda foram particularmente estudados em áreas de florestas densas: Floresta Amazônica (Kapos 1989; Malcolm 1994); Floresta Semidecidual (Tabanez et al. 1997; Paciência \& Prado 2004; Furusawa \& Cassino 2006); Floresta de Araucária (Fontoura et al. 2006), sendo poucos os estudos de efeitos de borda voltados para os cerrados, com exceção de Pivello et al. (1999a; b).

O presente estudo teve por objetivo testar a hipótese de que os efeitos de borda afetam alguns parâmetros vegetacionais e populacional em fragmentos de Cerrado. Para isso, estudou-se a estrutura da vegetação, o padrão de distribuição espacial de Vernonia aurea Mart. ex DC. (Asteraceae) e as condições microclimáticas em dois ambientes, borda e interior, de três fragmentos de Cerradão no sudoeste de Goiás.

\section{Material e métodos}

Caracterização do local de estudo - Foram estudados três fragmentos de Cerradão (fragmento 1: 1,5 ha; fragmento 2: 9 ha e fragmento 3: 35 ha) situados em 
regiões de interflúvio com altitude de cerca de 700-750 m (entre 16 $6^{\circ} 28^{\prime}-16^{\circ} 45^{\prime} \mathrm{S}$ e $51^{\circ} 78^{\prime}$ - $52^{\circ} 23^{\prime} \mathrm{W}$ ) no município de Caiapônia, sudoeste de Goiás, Brasil (Fig. 1). Os fragmentos estão localizados em propriedades rurais particulares e inseridos em uma matriz antropizada, composta por áreas desmatadas para fins de exploração agropecuária. Os fragmentos 1 e 2 estão localizados em áreas exploradas continuamente por práticas agrícolas (lavoura de soja, milho e sorgo), há pelo menos cinco anos, enquanto o fragmento 3 sempre esteve circundado por pastagens. Os três fragmentos estudados foram isolados em períodos próximos, porém em anos diferentes. $\mathrm{O}$ isolamento dos fragmentos 2 e 3 se deu no final da década de 1970 e, do fragmento 1 , início da década de 1980 (observação pessoal). Na região é comum encontrar fragmentos semelhantes aos estudados, de diferentes tamanhos, que são conservados com o objetivo de servirem, principalmente, de refúgio para o gado e/ou reservas legais das propriedades rurais, sendo denominados regionalmente por "capões de mato". Sendo assim, tais fragmentos estudados são, eventualmente, perturbados com atividades relacionadas ao uso e exploração do solo, como pastoreio, fogo, retirada seletiva de madeira para uso em construções rurais e lenha, pesticidas, dentre outros. Embora o uso do fogo para limpeza e revitalização de pastagens e palhadas de lavouras ainda ocorra na região, esta prática tem sido, gradativamente, deixada de ser utilizada pelos produtores rurais.

A fitofisionomia referente aos fragmentos pode ser classificada como uma formação florestal do tipo Cerradão pela predominância de um estrato arbóreo bem definido, com a presença de espécies arbóreas como Anadanantera macrocarpa, Bowdichia virgilioides, Cecropia spp., Dypterix alata, Hymenaea courbaril, Plathymenia reticulata, Qualea grandiflora e Xylopia aromatica, ocorrendo em conjunto com espécies arbustivas e herbáceas, como Alibertia edulis, Annanas spp., Curatela americana, Solanum lycocarpum e Vernonia spp. Segundo Ribeiro \& Walter (1998), a fitofisionomia do tipo Cerradão é uma formação florestal com aspectos xeromórficos e caracteriza-se pela presença de espécies que ocorrem tanto no Cerrado sentido restrito quanto na Mata Seca. O Cerradão possui um extrato arbóreo variando entre 8 e $15 \mathrm{~m}$ de altura, com dossel predominantemente contínuo e cobertura arbórea que pode variar de 50 a $90 \%$, proporcionando assim, condições de luminosidade que favorecem a formação de estratos arbustivo e herbáceo diferenciados (Ribeiro $\&$ Walter 1998).

De acordo com Dias (1992), a região apresenta solos profundos e bem drenados, com predomínio de Latossolos. O clima é do tipo quente semi-úmido com duas estações bem definidas: inverno seco e verão chuvoso (Nimer 1989). A fase seca, correspondente ao inverno, se estende de maio a setembro com índices

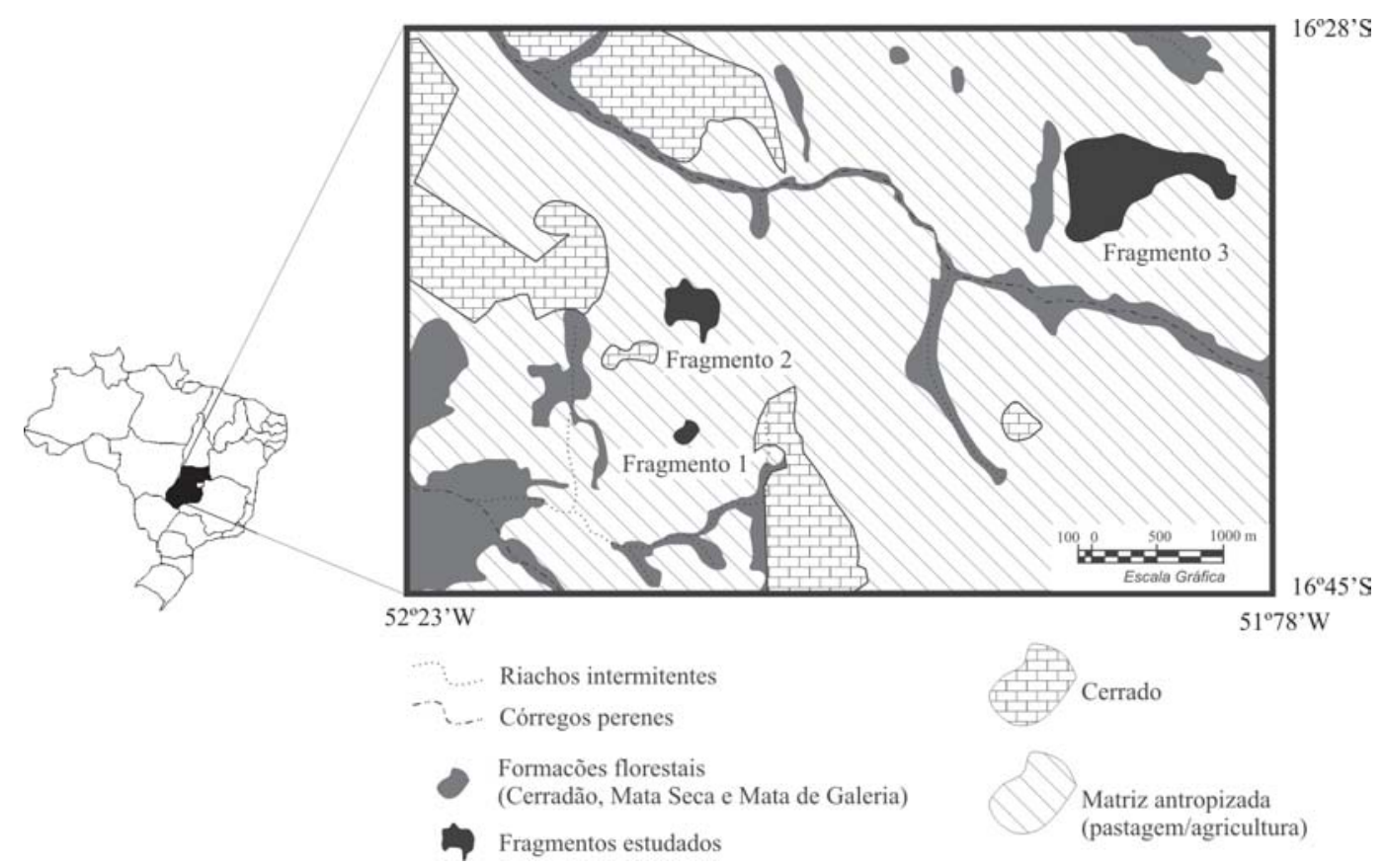

Figura 1. Fragmentos de Cerradão estudados em Caiapônia, GO, Brasil. 
pluviométricos inferiores a $60 \mathrm{~mm}$ mensais e temperatura média de $18^{\circ} \mathrm{C}$ e o verão chuvoso, com pluviosidade média acima de $180 \mathrm{~mm}$ mensais e temperatura média de $25^{\circ} \mathrm{C}$, estendendo-se pelo resto do ano (Pereira \& Freitas 1982; Nimer 1989).

Estrutura da vegetação - O inventário ocorreu na primeira quinzena de outubro/2004 (final da estação seca e início da estação chuvosa) e foi dividido em dois ambientes: borda e interior dos fragmentos. Esta classificação foi realizada considerando a forma e as medidas do menor fragmento (fragmento 1) e adotada, posteriormente, para os dois fragmentos maiores (fragmentos 2 e 3). Para tal, foi delimitado como ambiente de borda toda a faixa mais externa dos fragmentos igual à metade do raio referente ao menor diâmetro do fragmento 1 . Como o fragmento 1 possui forma elipsóide, com menor diâmetro de $60 \mathrm{~m}$, foi considerado ambiente de borda toda a faixa mais externa dos fragmentos com largura de $15 \mathrm{~m}$. A área restante foi considerada como o interior.

Para inferir a estrutura da vegetação nos dois ambientes, foram utilizados os seguintes parâmetros: circunferência do caule ao nível do solo; altura da planta e densidade de indivíduos. Para medir a circunferência do caule e a altura da planta, os indivíduos foram amostrados utilizando-se o método de quadrantes (Greig-Smith 1964; Müeller-Dombois \& Elemberg 1974). Foram demarcados, para cada fragmento, 60 pontos distribuídos em 10 linhas (5 linhas na borda e 5 no interior), distando $10 \mathrm{~m}$ entre pontos e, no mínimo, $30 \mathrm{~m}$ entre linhas. Para eliminar as espécies herbáceas e indivíduos juvenis, somente as plantas que apresentaram circunferência de caule ao nível do solo igual ou superior a $5 \mathrm{~cm}$ foram incluídas na amostragem. Os dados foram classificados em classes de frequiência de mesma amplitude e ordenados pela Análise de Componentes Principais (PCA), visando a visualização da distribuição das diferentes classes de circunferência do caule e altura da planta entre os ambientes de borda e interior, dentro e entre fragmentos (Legendre \& Legendre 1998). Essa abordagem estatística é importante para evidenciar tendências não visualizadas em testes de comparação entre médias, principalmente nos casos em que elas não se diferem estatisticamente.

A densidade de indivíduos foi obtida utilizando-se 10 parcelas (unidades amostrais) de $5 \times 5 \mathrm{~m}$, distribuídas, aleatoriamente, em cada fragmento (5 parcelas na borda e 5 no interior), onde foram quantificados todos os indivíduos com circunferência de caule ao nível do solo igual ou superior a $5 \mathrm{~cm}$.
As médias desses parâmetros para os ambientes de borda e interior dos três fragmentos foram comparadas duas a duas pelo teste $\mathrm{t}$ bi-caudal, ao nível de significância de 5\%. Como foram realizadas 15 comparações de seis médias diferentes tomadas par a par, utilizou-se o critério de Bonferroni para corrigir a probabilidade de Erro Tipo I.

Distribuição espacial - a influência dos efeitos de borda na estruturação populacional foi testada em populações de Vernonia aurea Mart. ex DC. (Asteraceae). Esta espécie foi utilizada por apresentar um número de indivíduos relativamente alto nos fragmentos estudados, distribuindo-se no interior e na borda. Segundo Joly (1979), as espécies de Vernonia ocorrem preferencialmente em ambientes típicos de vegetação aberta, como formações savânicas e campestres. Vernonia aurea é uma espécie de porte arbustivo ( 1,0 a $1,5 \mathrm{~m}$ de altura), comum em ambientes degradados (espécie pioneira) e considerada uma planta daninha em pastagens (Lorenzi 2000; Farias et al. 2002; Lara et al. 2003).

Devido ao porte herbáceo de $V$. aurea, foram demarcadas seis áreas de $6 \times 3 \mathrm{~m}$ em cada fragmento (três áreas no interior e três na borda), divididas, cada uma, em 200 parcelas (unidades amostrais) de $0,3 \times 0,3 \mathrm{~m}\left(0,09 \mathrm{~m}^{2}\right)$, totalizando $108 \mathrm{~m}^{2}$ de área amostrada em cada fragmento (metodologia proposta por Greig-Smith 1964). A delimitação dessas áreas, no interior e na borda, ocorreu aleatoriamente e seguiu a demarcação das faixas de ambientes (borda e interior) considerados para a estrutura da vegetação.

Para identificar a estrutura espacial da população, todos os indivíduos de $V$. aurea foram quantificados em cada parcela e a distribuição de freqüências observadas ( $\mathrm{n}^{\mathrm{o}}$ de parcelas com $0,1,2, \ldots, r$ indivíduos) foi comparada com frequiências esperadas. Os modelos teóricos utilizados para calcular as freqüências esperadas foram a Distribuição de Poisson (GreigSmith 1964) e a Distribuição Binomial Negativa (Ludwig \& Reynolds 1988; Krebs 1999).

A significância estatística foi obtida através do teste "G", onde é possível verificar o quanto a distribuição de freqüências observadas se ajusta à respectiva distribuição teórica (freqüências esperadas), com um certo número de graus de liberdade $(g . l .=q-2$, para o modelo de Poisson e g.l. $=q-3$, para o modelo Binomial Negativo, onde $q=r+1$, ou seja, o número de classes de frequiência com $0,1,2, \ldots, r$ indivíduos por parcela). Durante o cálculo de "G", as frequências esperadas menores que três e suas respectivas 
frequências observadas foram somadas, minimizando assim a probabilidade de erro tipo 1 (Sokal \& Rohlf 1995; Krebs 1999; Zar 1999).

Caso as freqüências observadas não difiram significantemente daquelas esperadas pelo modelo de Poisson, o padrão de distribuição espacial da população é aleatório. Caso contrário, a população não possui um padrão de distribuição aleatório ou esse padrão não foi detectado pelo teste (Greig-Smith 1964). Já o modelo Binomial Negativo testa a hipótese de que os indivíduos se distribuem de forma agregada em sua área de ocorrência (Krebs 1999). Se nenhuma dessas hipóteses forem aceitas ao nível de significância desejado, a hipótese de distribuição uniforme pode ser testada pela Distribuição Binomial Positiva (Ludwig \& Reynolds 1988).

Para obter o índice de dispersão dos indivíduos, foram selecionadas, aleatoriamente, dez amostras com 40 parcelas cada, tanto para o interior quanto para a borda dos fragmentos, visando minimizar a dependência entre as amostras (parcelas contíguas). Para cada amostra (sequiência com 40 parcelas), foi calculada a razão variância/média $(I)$, a qual mostra o quão agrupada se encontra a população (Ludwig \& Reynolds 1988; Krebs 1999). Se as freqüências observadas se distribuírem de acordo com o modelo de Poisson (distribuição espacial aleatório), significa que a média e a variância são iguais (Greig-Smith 1964) e portanto, espera-se que " $I$ " seja igual a 1 . Se " $I$ " for diferente de 1, a população apresenta distribuição agregada (" $I$ " $>1$ ) ou uniforme (" $I$ " $<1$; Ludwig \& Reynolds 1988). A significância dessa igualdade ( $I=1)$ foi testada pelo teste $\mathrm{t}$, com probabilidade uni-caudal e ao nível de significância de 5\%, utilizando-se as médias dos valores de " $I$ " para as seqüências selecionadas no interior e na borda dos fragmentos.

Parâmetros microclimáticos - Foram registradas a temperatura do ar, a umidade relativa do ar e a temperatura do solo. Esses dados foram coletados no mesmo período de amostragem dos parâmetros vegetacionais, em dez pontos no interior e dez na borda de cada fragmento, distribuídos aleatoriamente entre as faixas de ambientes consideradas para a estrutura da vegetação. Para comparação, essas variáveis também foram coletadas na matriz antropizada, numa faixa entre 5 e 20 metros afastando-se da borda dos fragmentos e com o mesmo número de réplicas. A temperatura e umidade relativa do ar foram mensuradas utilizando um Termo-Higrômetro digital MINIPA. A temperatura do solo foi obtida à profundidade de $2 \mathrm{~cm}$ abaixo da superfície, utilizando um termômetro com coluna de mercúrio. Para a coleta dos dados, foram selecionados, aleatoriamente, dez conjuntos com três pontos para cada fragmento, sendo cada conjunto composto por um ponto na matriz, um na borda e um no interior. Os parâmetros microclimáticos foram mensurados em horários diferentes (entre 8 e 17 h) para cada conjunto de pontos, mas no mesmo horário para os três pontos de um mesmo conjunto. O teste $\mathrm{t}$ bicaudal foi utilizado para comparação, par a par, entre as médias dos ambientes de borda e interior dos três fragmentos. Neste caso, foi utilizada a correção de Bonferroni para minimizar a probabilidade de Erro Tipo I.

\section{Resultados}

Estrutura da vegetação - Foram encontrados, no total, 40 indivíduos nas 15 parcelas $\left(375 \mathrm{~m}^{2}\right)$ localizadas no interior e 49 indivíduos na borda dos três fragmentos, com densidade média de 1.069 ind.ha ${ }^{-1}$ e 1.306 ind.ha $^{-1}$, respectivamente. Nos três fragmentos, a densidade média foi sempre maior na borda que no interior e com tendência de diminuir em direção aos fragmentos de maior tamanho (Tab. 1). Apesar disso, não houve diferença significativa entre a densidade de indivíduos em um mesmo ambiente (interior ou borda) nos fragmentos de diferentes tamanhos. A variância entre as densidades das parcelas (réplicas) em cada ambiente mostrou que as bordas dos três fragmentos possuem maior variação no número de indivíduos de um local para outro do que o interior (Tab. 1).

Um padrão geral também foi encontrado para a circunferência do caule e altura das plantas. Em todos os casos, esses parâmetros foram maiores no interior do que na borda dos fragmentos (Fig. 2). As Análises

Tabela 1. Densidade média de plantas em três fragmentos de Cerradão, Caiapônia, GO, Brasil. Médias seguidas de mesma letra não são diferentes ( $\mathrm{p}>0,05$; teste $\mathrm{t}$ bi-caudal, corrigido pelo critério de Bonferroni).

\begin{tabular}{lccc}
\hline Ambiente & \multicolumn{2}{c}{ Densidade média } & \multirow{2}{*}{ Variância } \\
\cline { 2 - 3 } & ind./25 m & ind./ha & \\
\hline Borda fragmento 1 & $3,34^{\mathrm{a}}$ & $1336^{\mathrm{a}}$ & 6,3 \\
Borda fragmento 2 & $3,38^{\mathrm{a}}$ & $1352^{\mathrm{a}}$ & 6,2 \\
Borda fragmento 3 & $3,08^{\mathrm{a}}$ & $1232^{\mathrm{a}}$ & 5,7 \\
Interior fragmento 1 & $2,80^{\mathrm{ab}}$ & $1120^{\mathrm{ab}}$ & 2,5 \\
Interior fragmento 2 & $2,62^{\mathrm{b}}$ & $1048^{\mathrm{b}}$ & 1,7 \\
Interior fragmento 3 & $2,60^{\mathrm{b}}$ & $1040^{\mathrm{b}}$ & 3,5 \\
\hline
\end{tabular}


de Componentes Principais mostraram tendências diferentes para os ambientes de borda e de interior dos fragmentos, sendo que os parâmetros analisados foram mais semelhantes dentro do que entre ambientes (Fig. 2, 3 e 4). No entanto, as bordas dos três fragmentos foram mais semelhantes entre si do que os ambientes de interior (Fig. 3 e 4). Os interiores dos fragmentos 2 e 3 foram mais semelhantes entre si do que com o interior do fragmento 1 quanto à circunferência do caule (Fig. 3), enquanto as bordas dos fragmentos 1 e 2 apresentaram plantas com alturas mais semelhantes entre si (indivíduos mais baixos) do que com aquelas encontradas na borda do fragmento
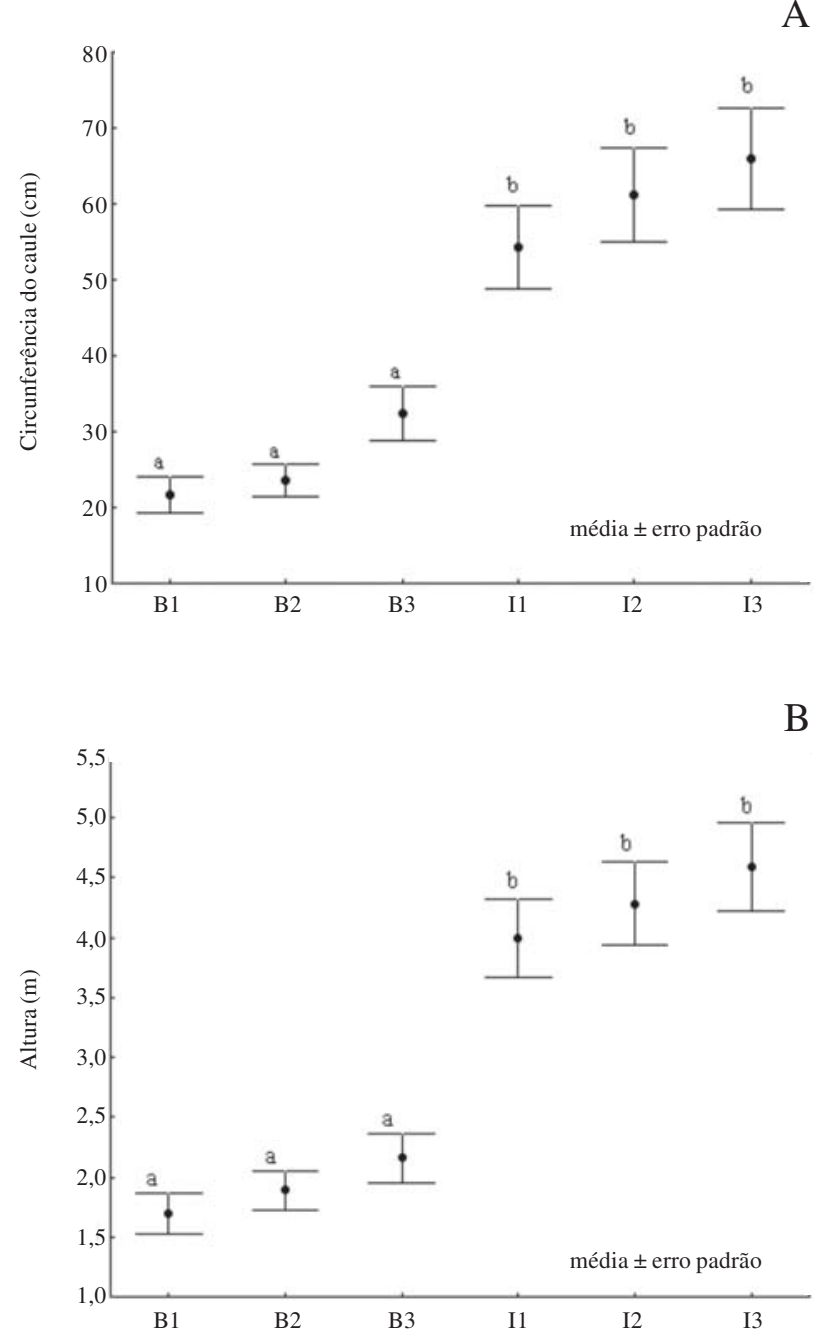

Figura 2. Médias de circunferência do caule (A) e de altura da planta (B) nos ambientes de borda e interior em três fragmentos de Cerradão, Caiapônia, GO, Brasil. As letras "B" e "I" indicam os ambientes de borda e interior dos fragmentos 1,2 e 3 , respectivamente ( $\mathrm{n}=60$ para cada média). Médias seguidas de mesma letra não são diferentes ( $p>0,05$; teste t bi-caudal, corrigido pelo critério de Bonferroni).
3 (indivíduos mais altos) (Fig. 4). Esses resultados não são visualizados na figura 2 , uma vez que os ambientes abordados (interior ou borda dos fragmentos) não diferem estatisticamente para os parâmetros utilizados (circunferência do caule e altura da planta). As figuras 2, 3 e 4 sugerem, em comum, que os ambientes de borda e interior diferem entre si para todos os fragmentos analisados e que os ambientes de interior estão mais relacionados com plantas de caule e altura maiores, enquanto os ambientes de borda apresentam plantas de caule e altura menores.

Distribuição espacial - Foram encontrados, no total, 2.057 indivíduos de $V$. aurea nos ambientes de interior e 3.610 nas bordas dos três fragmentos, com médias de 1,14 e 2,01 indivíduos por parcela, respectivamente. O número de indivíduos oscilou entre zero e sete nos ambientes de interior e de zero a nove indivíduos por parcela nas bordas dos fragmentos, rejeitando, em todos os casos, a hipótese de que os indivíduos se distribuem aleatoriamente na população, uma vez que os dados não se ajustaram à distribuição de Poisson (Tab. 2).

A hipótese de distribuição agregada foi confirmada através da Distribuição Binomial Negativa (Tab. 2). Os índices de dispersão (I) também confirmam essa hipótese, uma vez que os valores encontrados são, na maioria, maiores que 1 para o nível de significância de $5 \%$ (média do índice de dispersão para a borda $\mathrm{B} 1=2,00 ; \mathrm{B} 2=2,53$ e B3 = 1,93 - e interior $-\mathrm{I} 1=1,53$; $\mathrm{I} 2=1,84$ e $\mathrm{I} 3=1,05-$ dos fragmentos 1,2 e 3 , respectivamente). Em todos os casos, o valor médio do índice de dispersão foi maior na borda que no interior dos fragmentos.

Parâmetros microclimáticos - Os fragmentos apresentaram padrões semelhantes quanto à umidade e temperatura do ar e temperatura do solo entre os diferentes ambientes. As temperaturas do ar e do solo tendem a diminuir da matriz antropizada em direção ao interior dos fragmentos, enquanto a umidade relativa do ar apresenta comportamento oposto (Fig. 5). Apesar dos parâmetros microclimáticos apresentarem essa tendência geral, os dados revelam que os ambientes de borda e interior tendem a ser mais semelhantes entre si do que com a matriz antropizada (Fig. 5).

\section{Discussão}

A densidade de plantas com circunferência de caule igual ou maior que $5 \mathrm{~cm}$ não diferiu, em um mesmo ambiente (borda ou interior), entre os 
a borda do fragmento 3 (com 35 ha) mostrou-se diferenciada em comparação com os fragmentos $1 \mathrm{e}$ 2 (com 1,5 ha e 9 ha, respectivamente), possivelmente devido à influência do ambiente interno e da matriz circundante. Matrizes compostas por características diferentes também influenciam de forma diferente o recrutamento e estabelecimento de determinadas espécies vegetais (Nascimento et al. 2006). Assim, os diferentes tipos de matrizes relacionadas com os

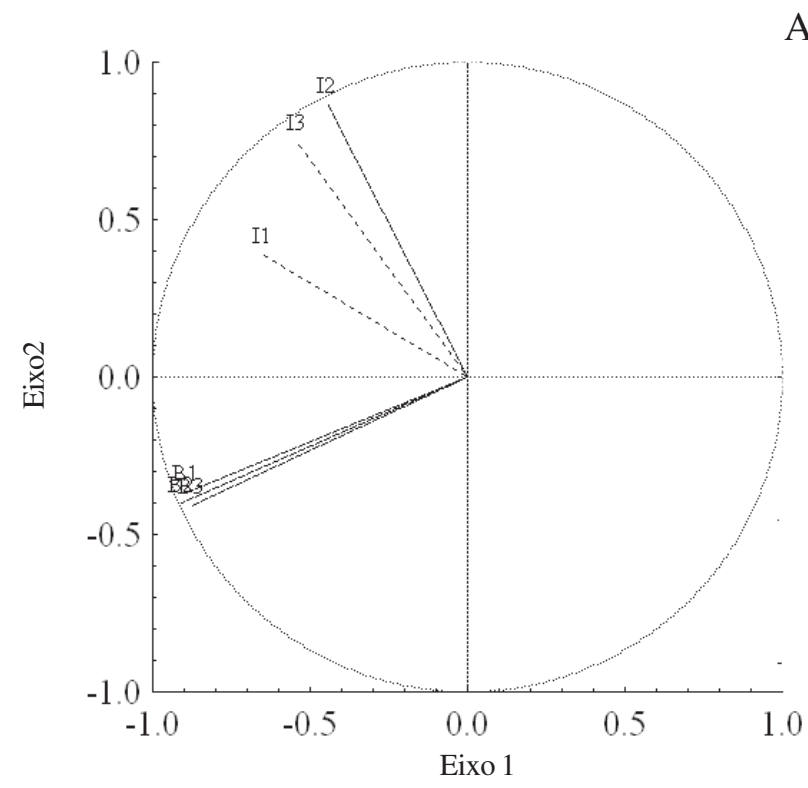

B

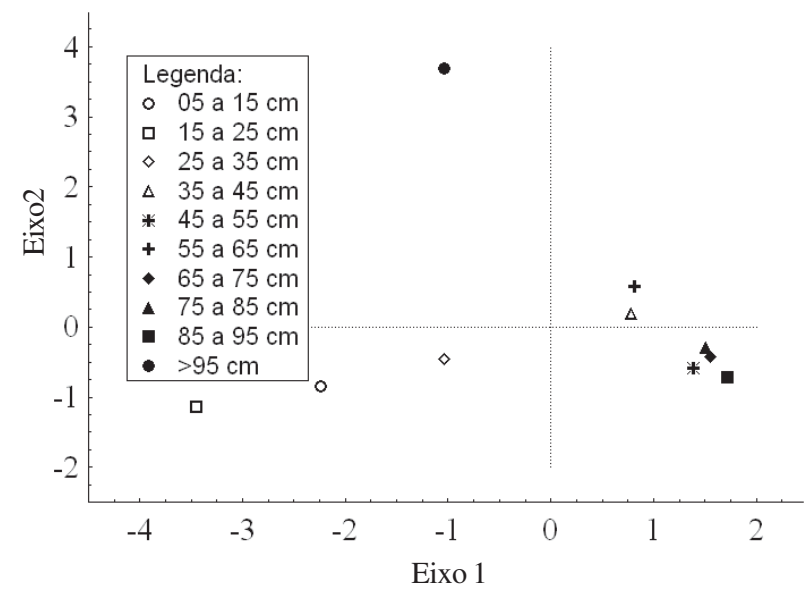

Figura 3. Análise de Componentes Principais (PCA) com os coeficientes de correlação de Pearson (A) e escores (B), evidenciando a distribuição das diferentes classes de circunferência do caule entre os ambientes de borda e interior em três fragmentos de Cerradão, Caiapônia, GO, Brasil. Em (A), as letras "B" e "I" indicam os ambientes de borda e interior dos fragmentos 1, 2 e 3 , respectivamente; em (B), as classes de frequiência estão fechadas à direita. O primeiro e o segundo eixo da PCA acumularam 55,4\% e 32,1\% da variância total, respectivamente. fragmentos 1 e 2 (matriz composta por lavoura há pelo menos cinco anos) e fragmento 3 (matriz composta por pastagem), podem influenciar o estabelecimento e consequentemente a composição de espécies nos três fragmentos, gerando padrões diferentes de altura das plantas como observado nesse estudo. Além disso, fragmentos maiores tendem a possuir maior riqueza de espécies animais (Laurance et al. 2002). Isso pode influenciar a dispersão de sementes de espécies
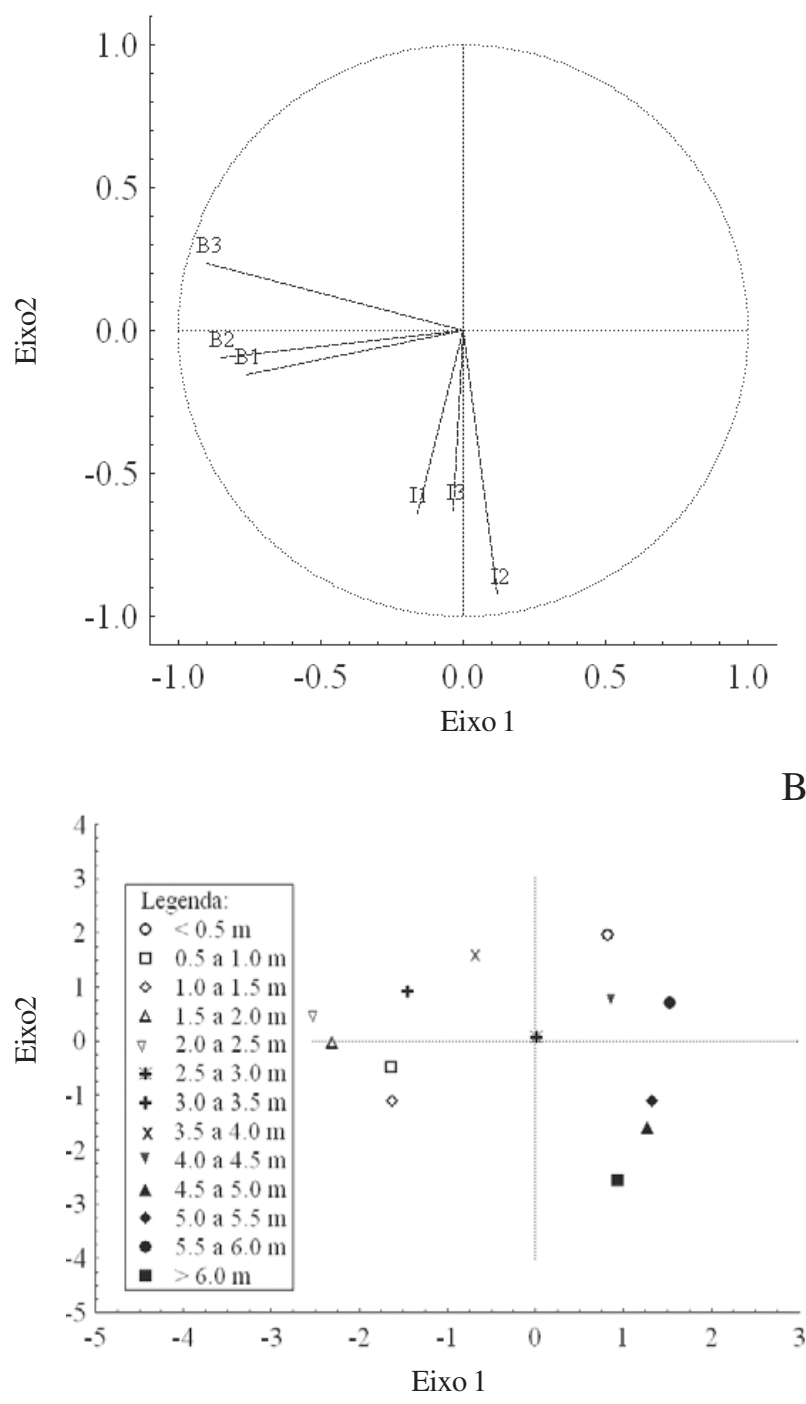

Figura 4. Análise de Componentes Principais (PCA) com os coeficientes de correlação de Pearson (A) e escores (B), evidenciando a distribuição das diferentes classes de altura da planta entre os ambientes de borda e interior em três fragmentos de Cerradão, Caiapônia, GO, Brasil. Em (A), as letras "B" e "I" indicam os ambientes de borda e interior dos fragmentos 1, 2 e 3, respectivamente; em (B), as classes de freqüência estão fechadas à direita. $\mathrm{O}$ primeiro e o segundo eixo da $\mathrm{PCA}$ acumularam $36,1 \%$ e 29,2\% da variância total, respectivamente. 
Tabela 2. Valores de G (teste de aderência) e suas respectivas probabilidades para comparação com as distribuições de freqüências esperadas pelos modelos de Poisson e Binomial Negativo para a borda e interior de três fragmentos de Cerradão, Caiapônia, GO, Brasil.

\begin{tabular}{|c|c|c|c|c|c|c|c|c|}
\hline \multirow[t]{2}{*}{ Fragmento } & \multicolumn{2}{|c|}{ Ambiente } & \multicolumn{3}{|c|}{ Poisson } & \multicolumn{3}{|c|}{ Binomial Negativo } \\
\hline & & & $\mathrm{G}$ & g.l. & $p$ & $\mathrm{G}$ & g.l. & $p$ \\
\hline \multirow[t]{6}{*}{1} & Borda & A1 & 50,80 & 5 & $<0,001$ & 1,88 & 6 & 0,930 \\
\hline & & $\mathrm{A} 2$ & 29,25 & 6 & $<0,001$ & 3,67 & 4 & 0,453 \\
\hline & & A3 & 21,92 & 5 & $<0,001$ & 4,81 & 5 & 0,440 \\
\hline & Interior & A1 & 26,13 & 4 & $<0,001$ & 6,34 & 4 & 0,175 \\
\hline & & $\mathrm{A} 2$ & 32,29 & 4 & $<0,001$ & 6,71 & 4 & 0,152 \\
\hline & & A3 & 11,98 & 4 & 0,017 & 4,02 & 3 & 0,259 \\
\hline \multirow[t]{6}{*}{2} & Borda & A1 & 34,91 & 6 & $<0,001$ & 2,67 & 5 & 0,751 \\
\hline & & $\mathrm{A} 2$ & 42,54 & 5 & $<0,001$ & 6,75 & 7 & 0,455 \\
\hline & & A3 & 33,67 & 7 & $<0,001$ & 5,64 & 5 & 0,343 \\
\hline & Interior & A1 & 13,75 & 4 & 0,008 & 10,66 & 5 & 0,059 \\
\hline & & A2 & 26,78 & 5 & $<0,001$ & 5,93 & 5 & 0,313 \\
\hline & & A3 & 15,99 & 5 & 0,007 & 7,12 & 4 & 0,130 \\
\hline \multirow[t]{6}{*}{3} & Borda & A1 & 25,98 & 6 & $<0,001$ & 3,56 & 4 & 0,469 \\
\hline & & $\mathrm{A} 2$ & 18,42 & 4 & 0,001 & 6,22 & 5 & 0,285 \\
\hline & & A3 & 34,46 & 4 & $<0,001$ & 5,93 & 3 & 0,115 \\
\hline & Interior & A1 & 9,48 & 4 & 0,050 & 9,23 & 4 & 0,055 \\
\hline & & A2 & 7,53 & 3 & 0,057 & 11,20 & 5 & 0,048 \\
\hline & & A3 & 13,27 & 3 & 0,004 & 4,59 & 2 & 0,101 \\
\hline
\end{tabular}

fragmentos com diferentes tamanhos, apesar de diminuir em direção aos fragmentos maiores. No entanto, a densidade foi altamente influenciada pelo ambiente em um mesmo fragmento. Nos três fragmentos analisados, a densidade foi significantemente maior nos ambientes de borda que no interior. Resultados semelhantes foram encontrados em fragmentos florestais na Amazonia central, onde a freqüente abertura de clareiras em áreas próximas às bordas (Camargo \& Kapos 1995) como resultado das altas taxas de mortalidade e danos às árvores (Ferreira \& Laurance 1997; Laurance et al. 1998a; D'Angelo et al. 2004), favorece o estabelecimento e crescimento de espécies pioneiras e secundárias adaptadas às condições de alta luminosidade (Nascimento \& Laurance 2006). Benitez-Malvido (1998) também demonstrou que a densidade de plântulas de espécies tardias declinou em locais próximos às bordas, indicando que o estabelecimento destas espécies vem diminuindo devido às condições diferenciadas relacionadas às áreas de borda.

Um padrão semelhante é relativamente visualizado nos dados gerados nesse estudo, uma vez que as plantas mais altas e com maior circunferência de caule estão localizadas no interior dos três fragmentos estudados, enquanto a borda está mais ligada às plantas baixas $\mathrm{e}$ com troncos mais delgados. Segundo Laurance et al. (1998a), um fator que pode determinar esse padrão observado é a diminuição na densidade de indivíduos de porte médio devido ao aumento das taxas de mortalidade de árvores de grande porte nas bordas dos fragmentos. Outro fator que também influencia é a taxa de recrutamento. De acordo com Laurance et al. (1998b), apesar da taxa de recrutamento se mostrar maior na borda que no interior dos fragmentos e em floresta contínua, a proporção de espécies tardias diminui em ambientes com altas taxas de recrutamento. Sendo assim, espera-se que os ambientes de borda apresentem plantas mais baixas e com caules mais delgados, determinados pela grande presença de indivíduos jovens de espécies pioneiras. A existência de temperaturas do ar e do solo mais elevadas e consequentemente menor umidade relativa na borda que no interior dos fragmentos também evidenciam a influência desses efeitos de borda.

Outro fator observado nesse estudo foi que os interiores dos fragmentos maiores são mais semelhantes entre si quanto à estrutura da vegetação, enquanto as bordas dos fragmentos menores é que se assemelham mais. $\mathrm{O}$ decréscimo na densidade de plantas em direção aos fragmentos de maior tamanho corroboram com essa hipótese, indicando que quanto menor o fragmento, maior é a influência dos parâmetros microclimáticos no interior em decorrência da maior proximidade com a borda, favorecendo a instalação e colonização de espécies pioneiras. Em contrapartida, 
A arbóreas tardias, prejudicando o estabelecimento de

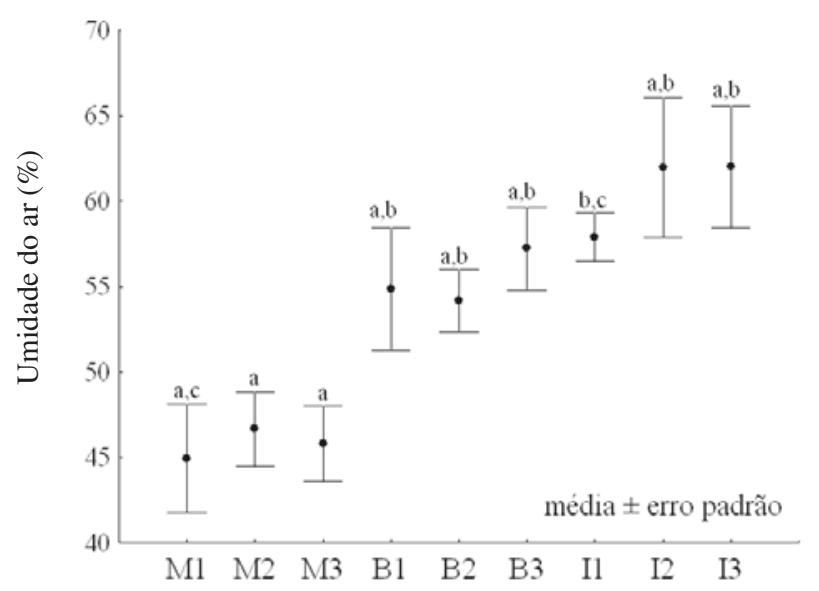

B

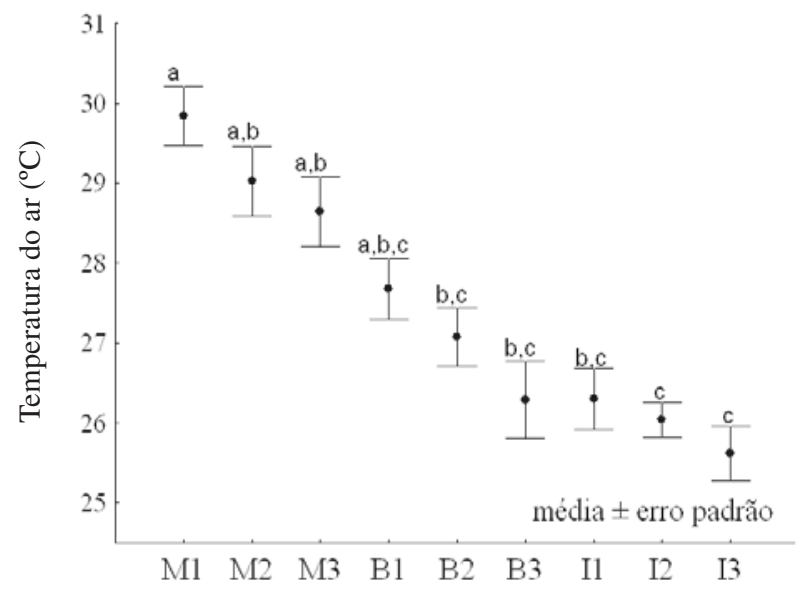

C

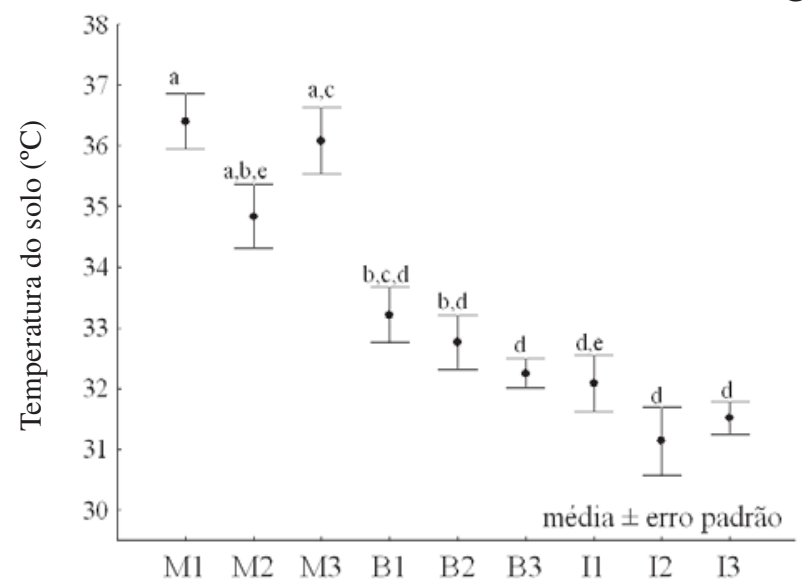

Figura 5. Médias de umidade do ar (A), temperatura do ar (B) e temperatura do solo (C) em três fragmentos de Cerradão, Caiapônia, GO, Brasil. As letras "M", "B" e "I" indicam os ambientes de matriz, borda e interior dos fragmentos 1,2 e 3 , respectivamente ( $\mathrm{n}=10$ para cada média). Médias seguidas de mesma letra não são diferentes ( $\mathrm{p}>0,05$; teste t bi-caudal, corrigido pelo critério de Bonferroni). plântulas dessas espécies nos ambientes de borda dos fragmentos menores (Benitez-Malvido 1998).

Em paisagens tropicais recentemente fragmentadas, os efeitos de borda têm um papel importante sobre a estrutura florestal, embora as escalas espaciais possam variar consideravelmente para diferentes alterações relacionadas aos efeitos de borda (Lovejoy et al. 1986; Didham \& Lawton 1999; Laurance et al. 2002). Para fragmentos de mesma forma, a relação área/perímetro é maior quanto maiores são os fragmentos e, portanto, quanto maior esta relação menor a intensidade dos efeitos de borda (Zudeima et al. 1996). Os fragmentos menores podem ser totalmente devastados por ventos fortes, enquanto que em fragmentos maiores podem ocorrer perturbações iniciais e posteriormente, estabelecimento de vegetação secundária em locais próximos às suas bordas (Gascon et al. 2000; Laurance et al. 2002).

Em paisagens fragmentadas como a deste estudo, em que os fragmentos florestais são frequentemente deteriorados a partir do uso de pesticidas, queimadas, mudanças microclimáticas e maior intensidade de ventos, além de interferir na composição e riqueza de espécies vegetais e animais (Laurance et al. 2002), esses efeitos de borda modificam a dinâmica e estrutura das populações, como verificado pelo padrão de distribuição espacial de $V$. aurea entre os diferentes ambientes e fragmentos. Apesar do padrão de distribuição espacial das populações de $V$. aurea revelar-se agregado em todos os fragmentos, os índices de dispersão indicam que os indivíduos no interior dos fragmentos estão menos agregados que aqueles da borda. Esse padrão de dispersão pode ser explicado considerando que os indivíduos da borda estão sujeitos a adversidades mais intensas (p.ex. maiores temperaturas do ar e do solo, menor umidade do ar, ventos e luminosidade com maior intensidade e frequiência ao longo do ano, maior evapotranspiração, maior exposição a pesticidas e ao fogo) que os indivíduos do interior. Os efeitos de borda envolvem mudanças na abundância e na distribuição de espécies, provocadas pelos fatores abióticos nas proximidades das bordas, como por exemplo, o aumento da densidade de plantas devido ao aumento da radiação solar (Murcia 1995). Dessa forma, espera-se que a competição interespecífica seja maior nesses ambientes, que segundo Greig-Smith (1964) leva as populações a apresentarem padrões espaciais cada vez mais agregados. Outra explicação para essa diferença nos padrões espaciais entre a borda e o interior dos 
fragmentos está ligada às condições microclimáticas de cada ambiente. Vernonia aurea, por ser pioneira e típica de vegetação aberta, como formações savânicas e campestres (Farias et al. 2002), pode sofrer alguma influência negativa quanto à colonização e dispersão para o interior do fragmento. Esse aspecto é notado tanto pelos índices de dispersão $(I)$ quanto pelo número de indivíduos encontrados, quase $35 \%$ menor no interior do que na borda do fragmento.

\section{Agradecimentos}

À Elenice S.L. Ribeiro e Edno Prado, pelo auxílio na coleta de campo; Divino Brandão, Luis Maurício Bini, Levi Carina Terribille e a um revisor anônimo, pelas sugestões ao manuscrito; à Capes, pelo auxílio financeiro por meio da bolsa de Mestrado.

\section{Referências bibliográficas}

Barbour, M.G.; Burk, J.H. \& Pitts, W.D. 1987. Terrestrial Plant Ecology. $2^{\text {nd }}$ ed. Califórnia, Benjamim/Cummings.

Benitez-Malvido, J. 1998. Impact of forest fragmentation on seedling abundance in a tropical rais forest. Conservation Biology 12: 380-389.

Camargo, J.L.C. \& Kapos, V. 1995. Complex edge effects on soil moisture and microclimate in central Amazonian Forest. Journal of Tropical Ecology 11: 205-221.

D’Angelo, S.A.; Andrade, A.C.S.; Laurance, S.G.; Laurance, W.F. \& Mesquita, R.C.G. 2004. Inferred causes of tree mortality in fragmented and intact Amazonian forests. Journal of Tropical Ecology 20: 243-246.

Davies-Colley, R.J.; Payne, G.W. \& Van Elswijk, M. 2000. Microclimate gradients across a forest edge. New Zealand Journal of Ecology 24: 111-121.

Dias, B.F.S. 1992. Cerrados: uma caracterização. Pp. 11-25. In: B.F.S. Dias (ed.). Alternativa de desenvolvimento dos cerrados: manejo e conservação dos recursos naturais renováveis. Brasília, IBAMA.

Didhan, R.K. \& Lawton, J.H. 1999. Edge structure determines the magnitude of changes in microclimate and vegetation structure in tropical forest fragments. Biotropica 31: 17-30.

Diniz, J.A.F. 1984. Geografia na agricultura. São Paulo, Difel.

Farias, R.; Alves, E.R.; Martins, R.C.; Barboza, M.A.; Zanenga-Godoy, R.; Reis, J.B. \& Rodrigues-da-Silva, R. 2002. Caminhando pelo cerrado: plantas herbáceoarbustivas, caracteres vegetativos e organolépticos. Brasília, EDUnB.

Fernandes, A. 1998. Fitogeografia Brasileira. Fortaleza, Ed. Multigraf.

Ferreira, L.V. \& Laurance, W.F. 1997. Effects of forest fragmentation on mortality and damage of selected tree in central Amazonia. Conservation Biology 20: 243-246.
Fontoura, S.B.; Ganade, G. \& Larocca, J. 2006. Changes in plant community diversity and composition across an edge between Araucaria forest and pasture in South Brazil. Revista Brasileira de Botânica 29: 79-91.

Forman, R.T.T. \& Godron, M. 1986. Landscape Ecology. New York, Wiley \& Sons.

Franklin, J.F. 1993. Preserving biodiversity: species, ecosystems, or landscapes? Ecological Applications 3: 202-205.

Furusawa, G.P. \& Cassino, P.C.R. 2006. Ocorrência e Distribuição de Calliphotidae (Díptera, Oestroidea) em um Fragmento de Mata Atlântica Secundária no Município de Engenheiro Paulo de Frontin, Médio Paraíba, RJ. Revista de Biologia e Ciências da Terra 6: 152-164.

Galetti, M.; Alves-Costa, C.P. \& Cazetta, E. 2003. Effects of forest fragmentation, anthropogenic edges and fruit color on the consumption of ornithocoric fruits. Biological Conservation 111: 269-273.

Gascon, C.; Williamson, G.B. \& Fonseca, G.A.B. 2000. Receding edges and vanishing reserves. Science 288: 1356-1358.

Greig-Smith, M.A.P. 1964. Quantitative plant ecology. $2^{\text {nd }}$ ed. London, Buther Worths.

Joly, A.B. 1979. Botânica: introdução à taxonomia vegetal. $5^{\text {a }}$ ed. São Paulo, Companhia Editora Nacional.

Kapos, V. 1989. Effects of isolation on the water status of forest patches in the Brazilian Amazon. Journal of Tropical Ecology 5: 173-185.

Kapos, V.; Wandelli, E.; Camargo, J.L. \& Ganade, G. 1997. Edge-related changes in environment and plant responses due to forest fragmentation in central Amazonia. Pp. 33-44. In: W.F. Laurance \& R.O. Bierregaard Jr. (eds.). Tropical Forest Remnants: ecology, management, and conservation of fragmented communities. Chicago, Chicago University Press.

Kershaw, K.A. 1973. Quantitative and dynamic plant ecology. $2^{\text {nd }}$ ed. New York, American Elsevier.

Kollmann, J. \& Buschor, M. 2003. Edge effects on seed predation by rodents in deciduous forests of northern Switzerland. Plant Ecology 164: 249-261.

Krebs, C.J. 1999. Ecological Methodology. $2^{\text {nd }}$ ed. Menlo Park, Benjamim/Cummings.

Lara, J.F.R.; Macedo, J.F. \& Brandão, M. 2003. Plantas Daninhas em Pastagens de Várzeas no Estado de Minas Gerais. Planta Daninha 21: 11-20.

Laurance, W.F.; Ferreira, L.V.; Rankin-de-Merona, J.M. \& Laurance, S.G. 1998a. Rain forest fragmentation and the dynamics of Amazonian tree communities. Ecology 79: 2032-2040.

Laurance, W.F.; Ferreira, L.V.; Rankin-de-Merona, J.M. \& Laurance, S.G. 1998b. Effects of forest fragmentation on recruitment patterns in Amazonian tree communities. Conservation Biology 12: 460-464.

Laurance, W.F.; Lovejoy, T.; Vasconcelos, H.L.; Bruna, E.M.; Didham, R.K.; Stouffer, P.; Gascon, C.; Bierregaard, R.; Laurance, S. \& Sampaio, E. 2002. Ecosystem decay of Amazonian forest fragments, a 22-year investigation. Conservation Biology 16: 605-618. 
Legendre, P. \& Legendre, L. 1998. Numerical ecology. $2^{\text {nd }}$ ed. Amsterdan, Elsevier Science.

Leite, E.J. 2001. Spatial distribution patterns of riverine forest taxa in Brasília, Brazil. Forest Ecology and Management 140: $257-264$.

Lorenzi, H. 2000. Plantas daninhas do Brasil: terrestres, aquáticas, parasitas e tóxicas. Nova Odessa, Instituto Plantarum.

Lovejoy, T.E.; Bierregaard, R.O.; Rylands, A.B.; Malcolm, J.R.; Quintela, C.E.; Harper, L.H.; Brown, K.S.; Powell, A.H.; Powell, G.V.N.; Schubart, H.O.R. \& Hays, M.B. 1986. Edge and other effects of isolation on Amazon forest fragments. Pp. 257-285. In: M.E. Soulé (ed.). Conservation Biology: The Science of Scarcity and Diversity. Massachusetts, Sinauer.

Ludwig, J.A. \& Reynolds, J.F. 1988. Statistical ecology: a primer on methods and computing. New York, John Wiley \& Sons.

Lundberg, S. \& Ingvarsson, P. 1998. Population dynamics of resource limited plants and their pollinators. Theorical Population Biology 54: 44-49.

Macdougall, A. \& Kellman, M. 1992. The understorey light regime and patterns of tree seedlings in tropical riparian forest patches. Journal of Biogeography 19: 667-675.

Malcolm, J.R. 1994. Edge effects in Central Amazonian forest fragments. Ecology 75: 2438-2445.

Mueller-Dombois, D. \& Ellenberg, H. 1974. Aims and methods of vegetation ecology. New York, John Wiley e Sons.

Murcia, C. 1995. Edge effects in fragmented forests: implications for conservation. Trends in Ecology and Evolution 10: 58-62.

Nascimento, H.E.M. \& Laurance, W.F. 2006. Efeitos de área e de borda sobre a estrutura florestal em fragmentos de floresta de terra-firme após 13-17 anos de isolamento. Acta Amazonica 36: 183-192.

Nascimento, H.E.M.; Andrade, A.C.S.; Camargo, J.L.C.; Laurance, W.F.; Laurance, S.G. \& Ribeiro, J.E.L. 2006. Effects of the Surrounding Matrix on Tree Recruitment in Amazonian Forest Fragments. Conservation Biology 20: $853-860$.

Nimer, E. 1989. Climatologia do Brasil. Rio de Janeiro, IBGE.

Paciência, M.L.B. \& Prado, J. 2004. Efeitos de borda sobre a comunidade de pteridófitas na Mata Atlântica da região de Una, sul da Bahia, Brasil. Revista Brasileira de Botânica 27: 641-653.

Pereira, R.F. \& Freitas, E.M. 1982. Climatologia. p. 626-644. In: PROJETO RADAMBRASIL, Folha SD-23-Brasília. v.29. Rio de Janeiro, IBAMA. (Levantamento de Recursos Naturais)

Pivello, V.R.; Carvalho, V.M.C.; Lopes, P.F.; Peccinini, A.A. $\&$ Rosso, S. 1999a. Abundance and distribution of native and alien grasses in a "Cerrado" (Brazilian Savanna) biological reserve. Biotropica 31: 71-82.
Pivello, V.R.; Shida, C.N. \& Meirelles, S.T. 1999b. Alien grasses in Brazilian savannas: a threat to the biodiversity. Biodiversity and Conservation 8: 1281-1294.

Primak, R.B. \& Rodrigues, E. 2001. Biologia da Conservação. Londrina, Midiograf.

Redding, T.E.; Hope, G.D.; Fortin, M.J.; Schmidt, M.G. \& Bailey, W.G. 2003. Spatial patterns of soil temperature and moisture across subalpine forest-clearcut edges in the southern interior of British Columbia. Canadian Journal of Soil Science 83: 121-130.

Ribeiro, J.F. \& Walter, B.M.T. 1998. Fitofisionomias do Bioma Cerrado. Pp. 89-168. In: S.M. Sano \& S.P. Almeida (eds.). Cerrado: ambiente e flora. Planaltina, EMBRAPA/CPAC. Ridley, M. 2006. Evolução. $3^{\text {a }}$ ed. Porto Alegre, Artmed.

Rizzini, C.T. 1997. Tratado de Fitogeografia do Brasil. São Paulo, Ed. Âmbito Cultural.

Rodrigues, P.J.F.P. \& Nascimento, M.T. 2006. Fragmentação Florestal: Breves Considerações Teóricas sobre Efeitos de Borda. Rodriguésia 57: 63-74.

Sala, O.E.; Chapin, F.S.; Armesto, J.J.; Berlow, E.; Bloomfield, J.; Dirzo, R.; Huber-Sanwald, E.; Huenneke, L.F.; Jackson, R.B.; Kinzig, A.; Leemans, R.; Lodge, D.M.; Mooney, H.A.; Oesterheld, M.; Poff, N.L.; Sykes, M.T.; Walker, B.H.; Walter, M. \& Wall, D.H. 2000. Global biodiversity scenarious for the year 2100. Science 287: 1770-1774.

Sokal, R.R. \& Rohlf, F.J. 1995. Biometry: the principles and pratic of statistics in biological research. $3^{\text {rd }} \mathrm{ed}$. New York, W.H. Freeman and Company.

Tabanez, A.A.J.; Viana, V.M. \& Dias, A.S. 1997. Conseqüências da fragmentação e do efeito de borda sobre a estrutura, diversidade e sustentabilidade de um fragmento de Floresta de Planalto de Piracicaba, SP. Revista Brasileira de Biologia 57: 47-60.

Viana, V.M.; Tabanez, A.A.J. \& Batista, J.L.F. 1997. Dynamics and restoration of forest fragments in the Brazilian Atlantic moist forest. Pp. 351-365. In: W.F. Laurance \& R.O. Bierregaard (eds.). Tropical Forest Remnants: Ecology, Management, and Conservation of Fragmented Landscape. Chicago, University of Chicago Press.

Wiens, J.A.; Stenseth, N.C.; Van Horne, B. \& Ims, R.A. 1993. Ecological mechanisms and landscape ecology. Oikos 66: 369-380.

Williams-Linera, G.; Domínguez-Gastelú, V. \& García-Zurita, M.E. 1997. Microenvironment and floristics of different edges in a fragmented tropical rainforest. Conservation Biology 12: 1091-1102.

Willson, M.F. \& Crome, F.H.J. 1989. Patterns of seed rain at the edge of a tropical Queensland rain forest. Journal of Tropical Ecology 5: 301-308.

Zar, J.H. 1999. Biostatistical analysis. $4^{\text {th }}$ ed. New Jersey, Prentiçe Hall.

Zudeima, P.A.; Sayer, J.A. \& Dijkman, W. 1996. Forest fragmentation and biodiversity: the case for intermediatesized conservation areas. Environmental Conservation 23: 290-297. 